\title{
Determination of the Optimally Efficient Sets in Special Classes of Graphs
}

\author{
Mihai TALMACIU \\ "Vasile Alecsandri” University of Bacau, \\ mtalmaciu@ub.ro
}

\begin{abstract}
A dominating set is said to be an efficient dominating set if, for every vertex $v \in V,|N[v] \cap S|=1[1]$. A set $S$ is called open irredundant if, for every vertex $\mathrm{u} \in \mathrm{S}$, there exists a vertex $\mathrm{v} \in \mathrm{V}-\mathrm{S}$ for which $\mathrm{N}(\mathrm{v}) \cap \mathrm{S}=\{\mathrm{u}\}$, in which case we say that $u$ efficiently dominates $v$. There exists a polynomial time algorithm for finding an optimally efficient set in an arbitrary graph. We determine directly the optimally efficient sets in confidentially connected graphs and unbreakable graphs. Also, we determine directly the open irredundant set, closed neighborhood packing set, the influence of a set in confidentially connected graphs and unbreakable graphs.
\end{abstract}

Keywords: Confidentially connected graphs, unbreakable graphs, open irredundant set, optimally efficient set.

\section{Introduction}

This article is motivated because there is only a polynomial time algorithm for finding an optimally efficient set in an arbitrary graph, and we determine it directly for special classes of graphs. Also, in [13] domination and irredundance parameters for some graphs have been extensively studied. For a survey see [9].

The efficiency of a set $\mathrm{S} \subseteq \mathrm{V}$ in a graph $\mathrm{G}=(\mathrm{V}, \mathrm{E})$, is defined as $\varepsilon(\mathrm{S})=\mid\{\mathrm{v} \in \mathrm{V}-\mathrm{S}$ : $|\mathrm{N}(\mathrm{v}) \cap \mathrm{S}|=1\} \mid$. The efficiency of a graph, denoted $\varepsilon(\mathrm{G})$, is defined to equal the maximum efficiency of a set $\mathrm{S} \subseteq \mathrm{V}$ in $\mathrm{G}$ [3]. A dominating set for a graph $G=(V, E)$ is a subset $D$ of $V$ such that every vertex not in $D$ is joined to at least one member of $D$ by some edge. That is, a set $D$ is a dominating set if $|N[v] \cap D| \geq 1$ for all vertices $v$ in $V(G)$. The domination number $\gamma(G)$ is the number of vertices in the smallest dominating set of $G$. A subset $S \subseteq V(G)$ is called a $k$-packing, if for any two distinct vertices $u, v$ in $S$, we have $d(u, v)>k$. A set $S$ is a closed neighborhood packing if for each $u$, $v \in S, u \neq v$ we have $N[u] \cap N[v]=\phi$. That is, a set $S$ is a closed neighborhood packing if $|N[v] \cap S| \leq 1$ for all vertices $v \in V(G)$. The packing number $\rho(G)$ is the size of the largest closed neighborhood packing. For all graphs $G$, $1 \leq \rho(G) \leq n$. The only graphs with $\rho(G)=n$ are graphs with no edges.

A dominating set $\mathrm{S}$ is called a perfect dominating set if every vertex $\mathrm{v} \in \mathrm{V}-\mathrm{S}$ is adjacent to exactly one vertex in S [4]. A dominating set is said to be an efficient dominating set if for every vertex $\mathrm{v} \in \mathrm{V}$,
$|\mathrm{N}[\mathrm{v}] \cap \mathrm{S}|=1$ [1]. The efficient domination number of a graph, denoted $F(G)$, is the maximum number of vertices that can be dominated by a set $S$ that dominates each vertex at the most once. A graph $G$ of order $n=$ $|V(G)|$ has an efficient dominating set if and only if $F(G)=n$.

A graph is efficient if and only if there exists an efficient dominating set. That is, a graph is efficient if and only if there exists a set $S$ which is both dominating and a closed neighborhood packing.

If a graph $G$ is efficient, then $\rho(G)=\gamma(G)$ [12] .

The influence of S is defined in [8] to be

$\mathrm{I}(\mathrm{S})=\sum_{v \in S}|N[v\}|=\sum_{v \in S}(1+\operatorname{deg}(v))$,

where $\operatorname{deg}(\mathrm{v})=|\mathrm{N}(\mathrm{v})|$, the cardinality of the open neighborhood of $\mathrm{v}$.

Thus, the efficient domination number of a graph $G$ is $F(G)=\max \{\mathrm{I}(\mathrm{S}): \quad S$ is a packing $\}=\max \left\{\sum_{v \in S}(1+\operatorname{deg}(v))\right.$

$: S \subseteq V(G)$ and $\mathrm{u}, \mathrm{v} \in \mathrm{S}$ implies $\mathrm{d}(\mathrm{u}, \mathrm{v})$ $\geq 3\}$. An $F(G)$-set $S$ is a set that is both a packing and $\mathrm{I}(\mathrm{S})=\mathrm{F}(\mathrm{G})$.

A set $\mathrm{S}$ is called open irredundant if for every vertex $\mathrm{u} \in \mathrm{S}$ there exists a vertex $\mathrm{v} \in \mathrm{V}-\mathrm{S}$ for which $\mathrm{N}(\mathrm{v}) \cap \mathrm{S}=\{\mathrm{u}\}$, in which case we say that $\mathrm{u}$ efficiently dominates $\mathrm{v}$ [7]. The upper open irredundance number, denoted $\operatorname{OIR}(G)$, equals the maximum number of vertices in an open irredundant set. Thus, $\operatorname{OIR}(G)$ equals the maximum number of vertices that can 
simultaneously and successfully broadcast a message in an Ethernet graph.

By contrast, the efficiency of a graph $\mathrm{G}$ equals the maximum number of vertices that can simultaneously receive a broadcast message in an Ethernet graph.

A set $\mathrm{S}$ is optimally efficient if two conditions are met: (i) for every vertex $\mathrm{v} \in \mathrm{V}-\mathrm{S}, \varepsilon(\mathrm{S} \cup$ $\{\mathrm{v}\}) \leq \varepsilon(\mathrm{S})$, and (ii) for every vertex $\mathrm{u} \in \mathrm{S}, \varepsilon(\mathrm{S}$ $-\{\mathrm{u}\})<\varepsilon(\mathrm{S})$.

In a network, the communication is said to be "confidential" if a message can be passed between any two vertices, without being intercepted by a third vertex. In the language of graph theory, this property stands for confidential connectivity.

There exists a polynomial time algorithm for finding an optimally efficient set in an arbitrary graph [10].

We determine directly the optimally efficient sets in confidentially connected graphs and unbreakable graphs.

Such communications are shown in [11].

\section{Notations and Fundamental Definitions}

Throughout this paper, $\mathrm{G}=(\mathrm{V}, \mathrm{E})$ is a simple (i.e. finite, undirected, without loops and multiple edges) graph [2]. Let co- $G=\bar{G}$ denote the complement graph of $\mathrm{G}$. For $\mathrm{U} \subseteq \mathrm{V}$ let $G(U)$ denote the subgraph of $G$ induced by $\mathrm{U}$. By G-X we mean the graph $\mathrm{G}(\mathrm{V}-\mathrm{X})$, whenever $\mathrm{X} \subseteq \mathrm{V}$, but we often denote it simply by $\mathrm{G}-\mathrm{v} .(\forall v \in \mathrm{V})$ when there is no ambiguity. If $v \in V$ is a vertex in $G$, the neighborhood $\mathrm{N}_{\mathrm{G}}(\mathrm{v})$ denotes the vertices of $\mathrm{G}-\mathrm{v}$ that are adjacent to v. We write $\mathrm{N}(\mathrm{v})$ when the graph $\mathrm{G}$ appears clearly from the context. The neighborhood of the vertex $\mathrm{v}$ in the complement of the graph $G$ is denoted by $\bar{N}(v)$. For any subset $\mathrm{S}$ of vertices in the graph $\mathrm{G}$ the neighborhood of $\mathrm{S}$ is $N(S)=\cup_{v \in S} N(v)-S$ and $\mathrm{N}[\mathrm{S}]=\mathrm{S} \cup \mathrm{N}(\mathrm{S})$.

A clique is a subset of $\mathrm{V}$ with the property that all the vertices are pairwise adjacent. The clique number (density) of $\mathrm{G}$, denoted by $\omega(\mathrm{G})$ is the cardinal of the maximum clique. A clique cover is a partition of the vertices set such that each part is a clique. $\theta(\mathrm{G})$ is the cardinal of a smallest possible clique cover of $\mathrm{G}$; it is called the clique cover number of G. A stable (or independent) set is a subset of $\mathrm{V}$ with the property that all the vertices are pairwise non-adjacent. The stability number of $G$ is $\alpha(\mathrm{G})=\omega(\bar{G})$; the chromatic number of $\mathrm{G}$ is $\chi(\mathrm{G})=\theta(\bar{G})$.

By $\mathrm{P}_{\mathrm{n}}, \mathrm{C}_{\mathrm{n}}, \mathrm{K}_{\mathrm{n}}$ we mean a chordless path on $n \geq 3$ vertices, the chordless cycle on $n \geq 3$ vertices, and the complete graph on $n \geq 1$ vertices. If $\mathrm{e}=\mathrm{xy} \in \mathrm{E}$, we also denote $\mathrm{x} \sim \mathrm{y}$; we also denote $\mathrm{x}^{\wedge} \mathrm{y}$ whenever $\mathrm{x}, \mathrm{y}$ are not adjacent in G. A set A is totally adjacent (non adjacent) with a set $\mathrm{B}$ of vertices $(\mathrm{A} \cap \mathrm{B}=\phi)$ if ab is (is not) edge, for any a vertex in $A$ and any $b$ vertex in $B$; we note denote $A \sim B(A+B)$. $A$ graph $G$ is $F$-free if none of its induced subgraphs is in $F$.

The subset $\mathrm{A} \subset \mathrm{V}$ is called a cutset if $\mathrm{G}-\mathrm{A}$ is not connected. If, in addition, none of the proper subsets of $A$ is a cutset, then $A$ is called a minimal cutset. The distance between any two vertices $\mathrm{u}, \mathrm{v} \in \mathrm{V}(\mathrm{G})$, denoted by $\mathrm{d}(\mathrm{u}, \mathrm{v})$, is the length of the shortest path from $u$ to $v$.

The paper is organized as follows. In Section 3 we give preliminary results. In Section 4 we give the results concerning confidentially connected graphs and unbreakable graphs.

\section{Preliminary Results}

At first, we recall the notion of a weak component.

Definition 1. ([14], [15], [16]) A set $A \subset V(G)$ is called a weak set of the graph $G$ if $N_{G}(A) \neq V(G)-A$ and $G(A)$ are connected. If $A$ is a weak set, maximal with respect to set inclusion, then $G(A)$ is called a weak component. For simplicity, the weak component $G(A)$ will be denoted by $A$.

Definition 2. ([14], [15], [16]) Let $G=(V, E)$ be a connected and non-complete graph. If $A$ is a weak set, then the partition $\{A, N(A), V$ $A \cup N(A)\}$ is called a weak decomposition of $G$ with respect to $A$.

The name of "weak component" is justified by the following result. 
Theorem 1. ([14],[15],[16]) Every connected and non-complete graph $G=(V, E)$ admits $a$ weak component A such that

$$
G(V-A)=G(N(A)+G(\bar{N}(A)) .
$$

Theorem 2. ([14], [15], [16]) Let $G=(V, E)$ be a connected and non-complete graph and $A \subset V$. Then $A$ is a weak component of $G$ if and only if $G(A)$ is connected and $N(A) \sim \bar{N}(A)$.

The next result, based on Theorem 1, ensures the existence of a weak decomposition in a connected and non-complete graph.

Theorem 3. If $G=(V, E)$ is a connected and non-complete graph, then $V$ admits a weak decomposition $(A, B, C)$, such that $G(A)$ is a weak component and

$G(V-A)=G(B)+G(C)$.

Theorem 2 provides an $O(n+m)$ algorithm for building a weak decomposition for a noncomplete and connected graph.

\section{Algorithm for the weak decomposition of a graph ([14], [16])}

Input: A connected graph with at least two nonadjacent vertices, $G=(V, E)$.

Output: A partition $V=(A, N, R)$ such that $G(A)$ is connected, $N=N(A), A \nsim R=\bar{N}(A)$. between any two vertices, without being intercepted by a third vertex. In the language of graph theory, this property stands for confidential connectivity.

At first, we recall the notions of weakly component and weakly decomposition.

Definition 3. ([14], [5], [6]) A graph $G=(V, E)$ with at least three vertices is called confidentially connected if $\forall(a, b, c) \in V^{3}$ three distinct vertices, there exists $P$ an $a, b$-path in $G$ such that $N[c] \cap V(P) \subseteq\{a, b\}$.

The confidential connectivity property can be translated as follows: every two vertices are joined by a path whose intersection with the neighborhood of any other third vertex is either empty or an extremity or both extremities of the path, that is

$\forall(a, b, c) \in V^{3}: N[c] \cap V\left(P_{a b}\right) \subseteq\{a, b\}$.

A new characterization of confidentially connected graphs, using weakly decomposition, is given below. Some similar results are stated in [14].

Theorem 4. A connected and non-complete graph $G=(V, E)$ is confidentially connected if and only if $\forall v \in V(G), \quad[\bar{N}(v)]_{G}$ is a weakly component.

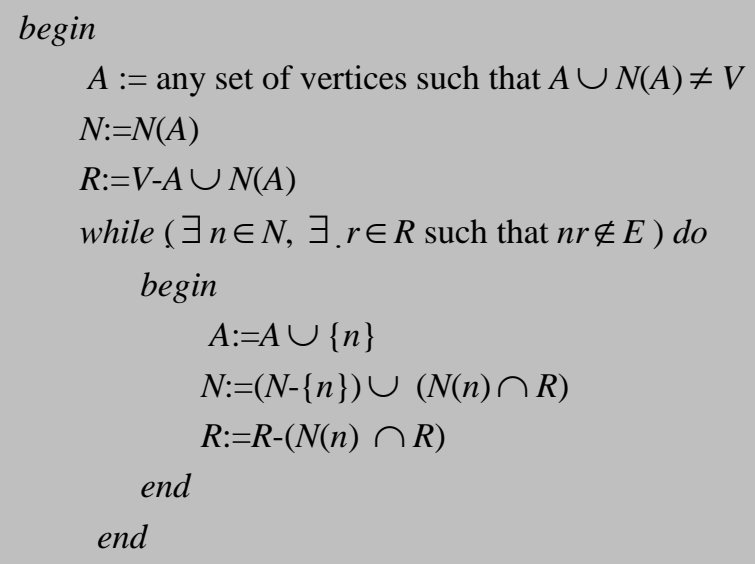

4. The Results Concerning Confidentially Connected Graphs and Unbreakable Graphs

\subsection{Basic properties}

In a network, the communication is said to be "confidential" if a message can be passed
Proof. Let $G$ be a confidentially connected graph and $\forall v \in V . \quad$ Because $N(v)=N(\bar{N}(v)), \quad \bar{N}(\bar{N}(v))=\{v\} \quad$ and $\{v\} \sim N(v)$ follow as $N(\bar{N}(v)) \sim \bar{N}(\bar{N}(v))$. Because $[\bar{N}(v)]_{G}$ is connected follow as $[\bar{N}(v)]_{G}$ is a weakly component of G. Let A 
be a weakly component of G. Considering the weakly decomposition procedure applied of $G$ with A the weakly component, in [10] follows that $\mathrm{R}$ has a single element. So any weakly component is non-neighborhood a vertex.

Vice versa. Let $\forall v \in V$ with $[\bar{N}(v)]_{G}$ weakly component. Because $[\bar{N}(v)]_{G}$ is weakly component follows as $[\bar{N}(v)]_{G}$ is connected. Because a weakly component is maximal with respect to set inclusion, for any two distinct vertices, their non-neighborhoods are not comparable. If there are two distinct vertices that are the extremities of an edge in $G$, with their neighborhoods comparable, then their non-neighborhoods would be comparable also, thereby contradicting the above remark. In [14] follows that $\mathrm{G}$ is confidentially connected.

Theorem 5 [14]. A connected and noncomplete graph $G=(V ; E)$ is unbreakable if and only if $[\bar{N}(v)]_{G}$ is a weak component of $G$ for $v$ in $V$ and $[\bar{N}(v)]_{\bar{G}}$ is a weak component of $\bar{G}$.

Definition 4. A graph $G$ is called minimal unbreakable if $G$ is unbreakable and none of its proper induced subgraphs is unbreakable.

Theorem 6 [14]. $G$ is minimal unbreakable if and only if $G$ is $C_{k}$ or $\bar{C}_{k}$ for some $k>=5$.

\subsection{The determination of the optimally efficient sets}

In this section we give two results that lead to the determination of an optimally efficient set and open irredundant in particular classes of graphs.

Theorem 7. Let $G=(V, E)$ be a connected and non-complete graph. If $G$ is confidentially connected graphs, the set $S=\{v\}$, for $v$ in $V$ with maxim degree, is an optimally efficient, open irredundant, closed neighborhood packing set and the influence of $S$ is the maxim degree of $G$.

Proof. Let $\mathrm{S}=\{\mathrm{v}\}, \forall v \in V$. We obtain $\varepsilon(\mathrm{S})=\operatorname{deg} \mathrm{G}(\mathrm{v})=$ the degree of $\mathrm{v}$. We rank the vertices after degrees. We obtain $\varepsilon(G)=\Delta(G)=$ the maximum degree of $\mathrm{G}$. Clearly, $S=\{v 0\}$, is open irredundant. Let $\mathrm{S}=\{\mathrm{v} 0\}$ such that $\varepsilon(S)=\varepsilon(G)$. Then $\forall w \in V-S, \quad \varepsilon(S \cup\{w\} \leq \varepsilon(S)$. Because $\forall u \in S, 0=\varepsilon(S-\{u\})<\varepsilon(S)$, because G, being connected, has no isolated vertices. So, $\mathrm{S}=\{\mathrm{v} 0\}$ is an optimally efficient set. $\mathrm{S}=\{\mathrm{v} 0\}$ is a closed neighborhood packing set, because

$$
\begin{aligned}
& \left|N[a] \cap\left\{v_{0}\right\}\right|=0, \forall a \in \bar{N}\left(v_{0}\right), \\
& \left|N[n] \cap\left\{v_{0}\right\}\right|=\left|\left\{v_{0}\right\}\right|=1, \forall n \in N\left(v_{0}\right) . \\
& I\left(\left\{v_{0}\right\}\right)=\Delta(G) .
\end{aligned}
$$

Calculating $\Delta$ and finding a vertex of maxim degree, Theorem 7 leads to a $\mathrm{O}$ (n) algorithm for determining the optimally efficient set, open irredundant set, closed neighborhood packing set in a confidentially connected graph.

From Theorem 5 and Theorem 7 follows:

Corollary 1. Let $G=(V, E)$ be a connected and non-complete graph. If $G$ is unbreakable, the set $S=\{v\}$, for $v$ in $V$ with maxim degree and set $T=\{w\}$, for $w$ in $V$ with minim degree, is an optimally efficient, open irredundant, closed neighborhood packing set, the influence of $S$ is the maxim degree of $G$ and the influence of $T$ is the minim degree of $G$.

Calculating $\Delta$ and $\delta$ (the maximum degree of $\mathrm{G}$ ) and finding a vertex of maxim degree and a vertex of minimum degree, Corollary 1 leads to a $\mathrm{O}(\mathrm{n})$ algorithm for determining the optimally efficient set, open irredundant set, closed neighborhood packing set in a is unbreakable graph.

Theorem 8. Let $G=(V, E)$ be a connected and non-complete graph with $n$ vertices. If $G$ is minimal unbreakable then the set

$$
S=\left\{3 k+1 \mid 0 \leq k \leq 3\left[\frac{n}{3}\right]-2\right\}
$$

is an optimally efficient set, open irredundant set, closed neighborhood packing set in $G$ or in $\bar{G}$.

Proof. If $\mathrm{G}$ is minimal unbreakable then $\mathrm{G}$ is $C_{n}$ or $\bar{C}_{n}$. Let $\mathrm{V}(\mathrm{G})=\{1,2, \ldots, \mathrm{n}\}$ be such that, taking any two consecutive vertices that are adjacent, we obtain that

$$
S=\left\{1,4, \ldots, 3\left[\frac{n}{3}\right]-2\right\}
$$


is an optimally efficient set. Indeed, the vertices in $\mathrm{V}$-S have exactly one neighbor in $\mathrm{S}$, $\varepsilon(S)=|V-S|=n-\left[\frac{n}{3}\right]$, clearly

$\varepsilon(S \cup\{v\} \leq \varepsilon(S), \forall v \in V-S$,

$\varepsilon(S-\{u\})<\varepsilon(S), \forall u \in S$.

Because $\forall u \in S, \exists u+1 \in V-S$ such that $N(u+1) \cap S=\{u\} \quad$ follow $\mathrm{S}$ is open irredundant set. Because $|N[v] \cap S| \leq 1, \forall v \in V$ follow $\mathrm{S}$ is closed neighborhood packing set.

\section{Conclusions and Future Work}

In this paper we determine the optimally efficient sets in confidentially connected graphs and unbreakable graphs.. Our future work will determine the optimally efficient sets in other classes of graphs.

\section{Acknowledgment}

The research was supported by the project entitled Classes of graphs, complexity of problems and algorithms, "AR-FRBCF”, 20122013, a Bilateral Cooperation Research Project, involving Romanian Academy, National Academy of Sciences of Belarus, Republican Foundation for Fundamental Research and "Vasile Alecsandri" University of Bacau.

\section{REFERENCES}

1. BANGE, D. W., A. E. BARKAUSKAS, P. J. SLATER, Efficient Dominating Sets in Graphs, in: R. D. Ringeisen, F. S. Roberts (Eds.), Applications of Discrete Mathematics, SIAM, Philadelphia, PA, 1988, pp. 189-199.

2. BERGE, C., Graphs, North-Holland, Amsterdam. 1985.

3. BERNHARD, P. J., S. T. HEDETNIEMI, D. P. JACOBS, Efficient Sets in Graphs, Discrete Applied Mathematics, vol. 44, 1993, pp. 99-108.

4. COCKAYNE, E. J., B. L. HARTNELL, S. T. HEDETNIEMI, R. LASKAR, Perfect Domination in Graphs, Journal of
Combinatorics, Information \& System Sciences, vol. 18, 1993, pp. 136-148.

5. CROITORU, C., E. OLARU, M. TALMACIU, Confidentially Connected Graphs, The annals of the University "Dunarea de Jos" of Galati, Proceedings of the international conference "The risk in contemporany economy", Supplement to Tome XVIII (XXII), 2000.

6. CROITORU, C., M. TALMACIU, On Confidentially Connected Graphs, Buletinul Stiintific al Universitatii din Baia Mare. Seria B, Matematica - Informatica, vol. XVI, Nr. 1, 2000, pp. 13-16.

7. FARLEY, A. M., N. SCHACHAM, Senders in Broadcast Networks: Open Irredundancy in Graphs, Congressus Numerantium, vol. 38, 1983, pp. 47-57.

8. GRINSTEAD, D. L., P. J. SLATER, Fractional Domination and Fractional Packing in Graphs, Congressus Numerantium, vol. 71, 1990, pp. 153-172.

9. HEDETMIEMI, S. M., S. T. HEDETMIEMI, R. REYNOLDS, Combinatorial Problems on Chessboards: II, In T. W. Haynes, S. T. Hedetniemi, and P. J. Slater, editors, Domination in Graphs: Advanced Topics, Marcel Kekker, Inc. 1997.

10. HEDETNIEMI, S. M., S. T. HEDETNIEMI, H. JIANG, K. E. KENNEDY, A. A. McRAE, A Selfstabilizing Algorithm for Optimally Efficient Sets in Graphs, Information Processing Letters, vol. 112, 2012, pp. 621-623.

11. PAGE, T., An Artificial Neural Network (ANN) in Electronic Product Design, Studies in Informatics and Control, vol. 21(3), 2012, pp. 259-266. ISSN 1220-1766

12. RUBALCABA, R. R., A. SCHNEIDER, P. J. SLATER, A Survey on Graphs which Have Equal Domination and Closed Neighborhood Packing Numbers, AKCE International Journal of Graphs and Combinatorics., vol. 3, No. 2, 2006, pp. 93-114.

13. SINKO, A. P. J. SLATER, An Introduction to Influence Parameters for 
Chessboard Graphs, Congressus Numerantium, vol. 172, 2005, pp. 15-27.

14. TALMACIU, M., Decomposition Problems in the Graph Theory with Applications in Combinatorial Optimization - Ph. D. Thesis, University "Al. I. Cuza" Iasi, Romania, 2002.
15. TALMACIU, M., E. NECHITA, Recognition Algorithm for Diamond-free Graphs, Informatica, vol. 18, 2007, pp. 457-462.

16. TALMACIU, M., E. NECHITA, G. C. CRISAN, A Recognition Algorithm for a Class of Partitionable Graphs that Satisfies the Normal Graph Conjecture, Studies in Informatics and Control, vol. 18, 2009, pp. 349-354. 